\title{
Multi-fractal-interslipface Angle Curves of a Morphologically Simulated Sand Dune
}

\author{
B. S. DAYA SAGAR* \\ Centre for Remote Sensing and Information Systems, Department of Geoengineering, \\ Andhra University, Visakhapatnam- 530003 , India
}

(Received 25 March 2000)

\begin{abstract}
A sand dune is simulated by means of a non-linear mathematical morphological transformation of which the fractal dimensions with corresponding interslipface angles are computed. This exercise has relevance to test the validity of the model by considering various time series sand dune data that can be retrieved from the robust satellite remote sensing sensors.
\end{abstract}

Keywords: Sand dune; Mathematical morphology; Fractal dimension; Interslipface angle

In this communication, a supply point of the sand that acts as anitiator that receives the accumulated sand due to interferential wind forces forms the symmetrical sand dunes. This process is simulated by applying iterative morphological dilation by following a non-linear morphological transformation with respect to a generator which is also represented in morphological terms that posses certain characteristic information like an origin direction and orientation. In order to simulate the symmetrical sand dune in discrete space, an inverted triangular type of generator with center as origin and bottom as the vertices is used. At each iteration the fractal dimension has been computed and at the $n$th generation of the simulation the fractal dimension is found to be converged to the value 1 in two dimensional case. This has been represented in a multifractal curve by a graphical plot between the fractal dimensions at respective iteration of morphological dilation and the degree of multiscale dilations. This rapid communication contains three brief parts. The first part gives a brief introduction to the multiscale morphological dilation [1]. In the second part the simulated sand dune with the results are presented both mathematically and diagramatically for a better understanding of the simulated model and concluded with a further scope from the remote sensing perspective.

Dilation $(\delta)$, besides erosion, opening and closing [1], is one of the simplest quantitative

\footnotetext{
* Present address: Centre for Remote Imaging, Sensing, Processing (CRISP), Faculty of Science, National University of Singapore, Lower Kent Ridge Road, Singapore 119260. e-mail: crsbsds@nus.edu.sg, bsdsagar@hotmail.com
} 
morphological set transformations. The discrete binary image, $M$, is defined as a finite subset of Euclidean two dimensional space, $l R^{2}$ that can admit values between 1 and 0 . Hereafter the initiator refers to as $M$ that is a supply point in the present context. Prior to understand the simulation process morphological dilation is defined as a set transformation that expand a set in a meaningful way. This morphological transformation can be visualized as working with two images. They are initiator $M$, and a generator that is represented as $S$. Each $S$ has a designed shape that can be thought of as a probe of the $M$. This basic morphological transformation, i.e., dilation besides erosion, and opening and closing can be defined with respect to the generator $S$ with scaling factor $e$, supply point $M$. The multiscale morphological dilation is mathematically represented as follows.

Dilation : $\delta_{s}^{e}(M)=\left\{m: S_{m} \cap M\right\}=\cup_{s \in S} M_{s}$ where $e=1,2, \ldots, n$ where $S=(s: s \in S)$, i.e., $S$ rotated $180^{\circ}$ around the origin and $S \neq$ transpose of $S$ in this context, then the morphological dilation is dissimilar to Minkowski addition as is the case when $S=$ transpose of $S$.

This transformation is iteratively performed on the supply point, in other words an initiator, a dot in the two dimensional space to have different degrees of conical sand dunes with different peaks by means of a generator which is in an inverted triangle in shape. In contrast the initiator is the supply area depicted as a base with stationary length in the earlier work [2]. Due to the simplicity in description the shape of the generator at different scales is not described diagramatically. Figure 1 gives a consolidated diagram that consists of coded conical sand dune and overlaid systematically (upto 25 iterative dilations of initiator by a generator). This simulated conical sand dune, which is exactly similar to the one simulated by Bertz et al. [3], also consists of the simulated conical sand dune at

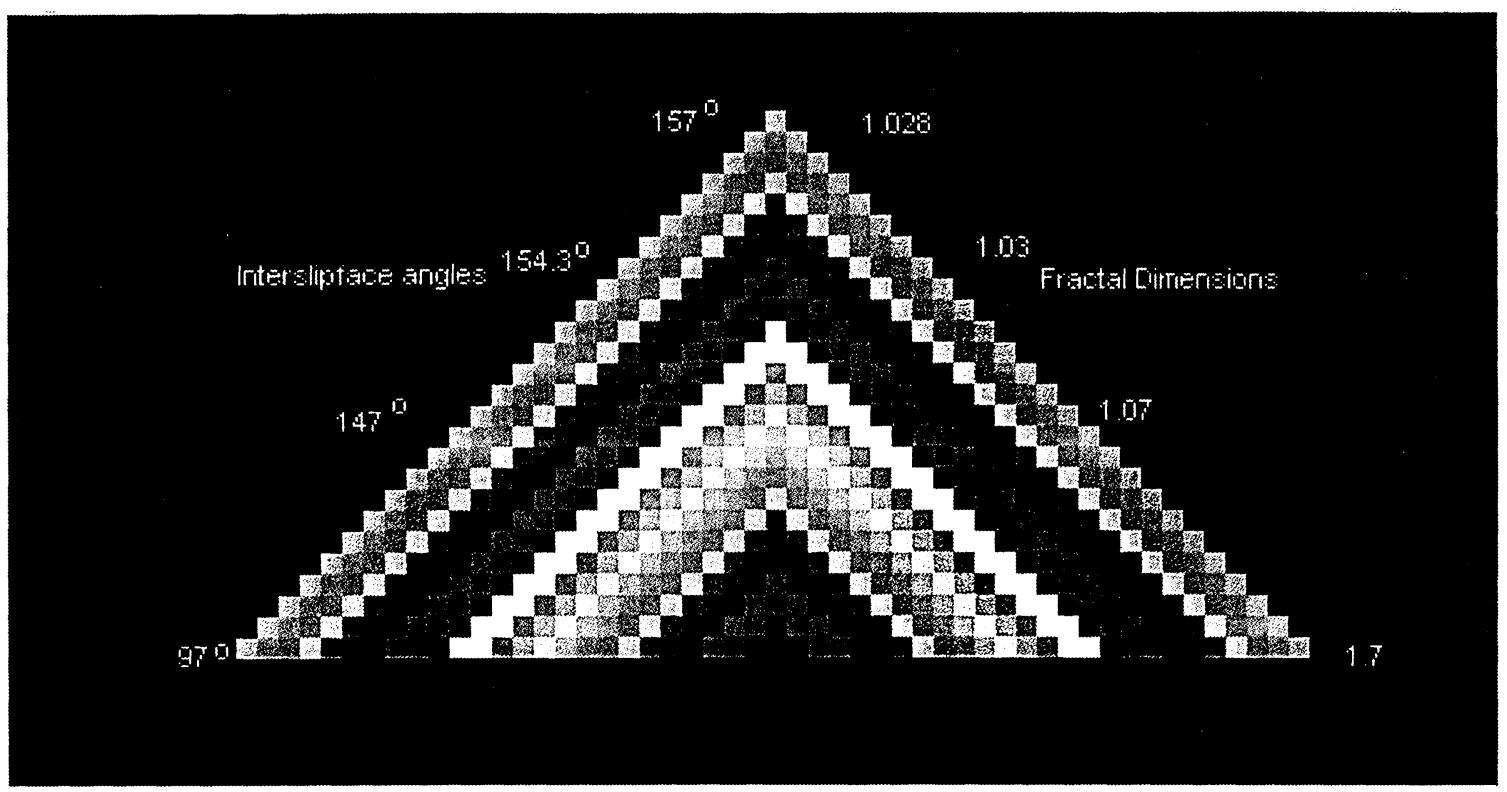

FIGURE 1 The sand dune shown is the digital image simulated from supply point (initiator) and a generator (inverted triangle) with center as origin and bottom as vertices. Simulation of sand dune is shown at different degrees of morphological dilations of initiator by generator. The fractal dimensions at the respective growth levels of Sand dune are shown at the right side of the axis. At the left, the interslipface angles have been depicted for respective degree of sand dune growth. To have a visual appeal, the simulated sand dune is enlarged. Hence the diagram at the high iterates of dilations are protruding, in contrast to the interslipface angles mentioned. 
respective degree of growth of sand dunes. Further a graphical plot is shown between the dilation degree and the corresponding fractal dimensions of sand dunes at the corresponding degree of dilations in Figure 2a. This is done to have a visual appeal to understand what author intends to say. The interslipface angle [2] at corresponding level of sand dune in the growth process simulated through multiscale dilations has been computed by following Eq. (2) proposed by Sagar [2].

$$
\alpha=\{\log N / \log [2 \operatorname{Sin}(\theta / 2)]\}-D_{T}
$$

where $\alpha+D_{T}=D ; N=2(2$ slipfaces for the sand dune)

From Eq. (2), it is reduced to compute the interslipface angles $(\theta)$ by incorporating the

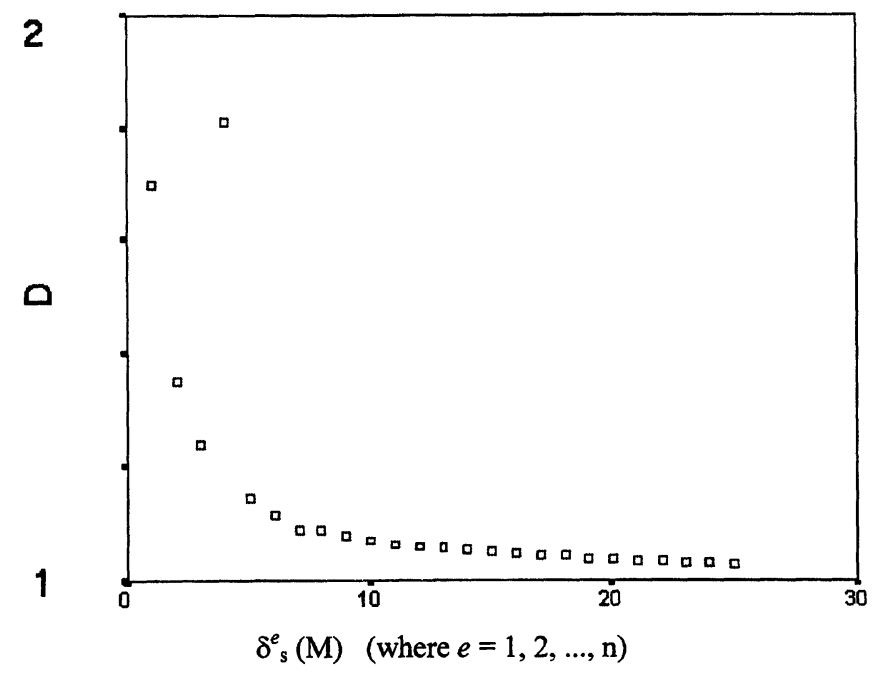

FIGURE 2a A multi-fractal curve of the simulated conical sand dune.

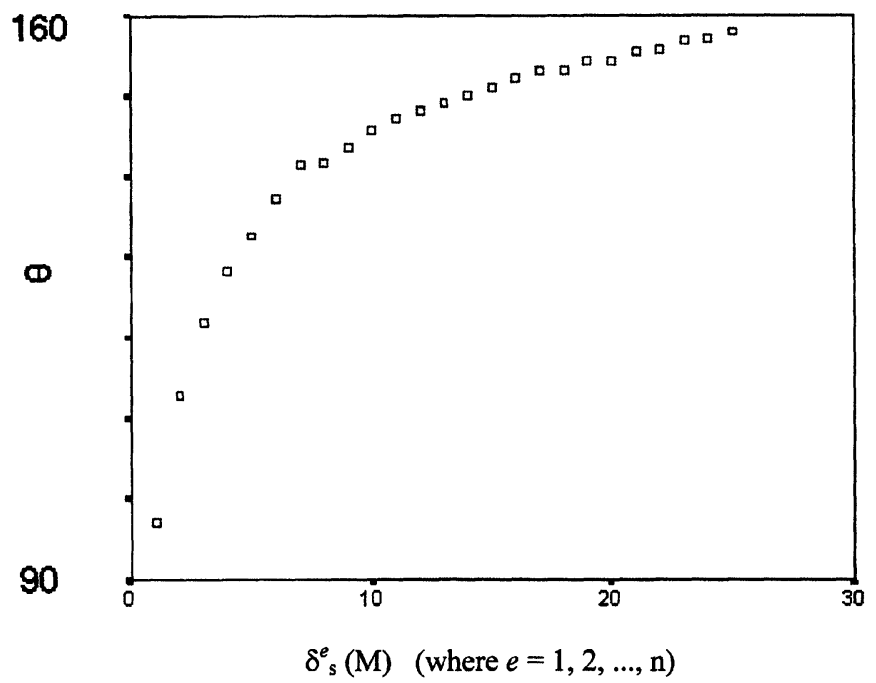

FIGURE 2b A multi-interslipface angle curve of the simulated conical sand dune. 
TABLE I Fractal dimensions and corresponding interslipface angles

\begin{tabular}{lcc}
\hline Dilation degree & Fractal dimension & $\begin{array}{c}\text { Interslipface angle } \\
\text { in degrees }\end{array}$ \\
\hline 1.00 & 1.70 & 97.17 \\
2.00 & 1.35 & 112.94 \\
3.00 & 1.24 & 122.05 \\
4.00 & 1.81 & 128.32 \\
5.00 & 1.14 & 132.74 \\
6.00 & 1.11 & 137.20 \\
7.00 & 1.09 & 141.40 \\
8.00 & 1.09 & 141.59 \\
9.00 & 1.08 & 143.59 \\
10.00 & 1.07 & 145.75 \\
11.00 & 1.06 & 147.14 \\
12.00 & 1.06 & 148.11 \\
13.00 & 1.06 & 149.07 \\
14.00 & 1.05 & 150.00 \\
15.00 & 1.05 & 151.05 \\
16.00 & 1.05 & 152.14 \\
17.00 & 1.04 & 152.95 \\
18.00 & 1.04 & 153.04 \\
19.00 & 1.04 & 154.29 \\
20.00 & 1.04 & 154.29 \\
21.00 & 1.03 & 155.45 \\
22.00 & 1.03 & 155.62 \\
23.00 & 1.03 & 156.69 \\
24.00 & 1.03 & 157.75 \\
25.00 & 1.03 & 157.80 \\
\hline & &
\end{tabular}

number of slipfaces ( $N=2$ in the present context, and the normalized fractal dimension $(\alpha)$ ). Now this reduced equation is as follows.

$$
\theta=2 \operatorname{Sin}^{-1}\{[10 \exp \cdot(\log N / D)] / 2\}
$$

The computed fractal dimensions $(D)$ and corresponding interslipface angles $(\theta)$ have been depicted in Table I. From the study, a multifractal and multi-interslipface angle curves are plotted
(Fig. 2a, b), between the degree of dilation, and fractal dimensions [4] as well as corresponding interslipface angles. The interslipface angle limits are between $97^{\circ}$ and $157^{\circ}$, of which the corresponding fractal dimensions range between 1.7 and 1 for this morphologically simulated sand dune growth. It is worth mentioned here, as the base length of the sand dune increases, the dune becomes flattened in this simulated sand dune model. These results are sensitive with a variation in any of the characteristic information such as origin, orientation, direction of the generator that plays a vital role in the simulation process. With this initial information and type of sand dune, morphological evolution can be quantified precisely to understand the nature or phenomenon of self organized criticality. With the advent of recent advances in the generation of high resolution digital elevation models from the remotely sensed data, the scope of the present study is that it can test the validity of this multi-fractal-interslipface angle curves by studying the natural conical sand dunes which are predominant in Central Asia (e.g., Bharkan sand dunes).

\section{References}

[1] Serra, J. (1982). Image analysis and mathematical morphology, Academic Press, London.

[2] Sagar, B. S. D. (1999). Morphological evolution of a pyramidal sandpile through bifurcation theory: a qualitative model, Chaos, Solitons, Fractals, 10(9), 1559-1566.

[3] Bretz, M., Cunningham, J. B., Kurczynsky, P. L. and Nori, F. (1992). Imaging of avalanches in granular materials, Physical Review Letters, 69, 2431.

[4] Mandelbrot, B. B. (1982). Fractal geometry of nature, San Francisco, Freeman \& Co., p. 468. 


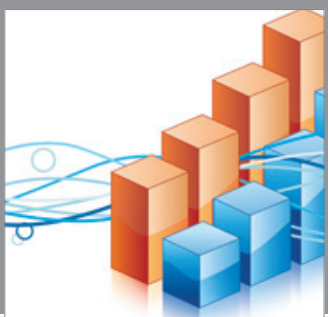

Advances in

Operations Research

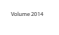

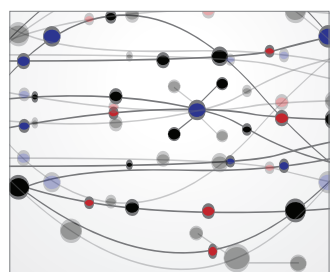

\section{The Scientific} World Journal
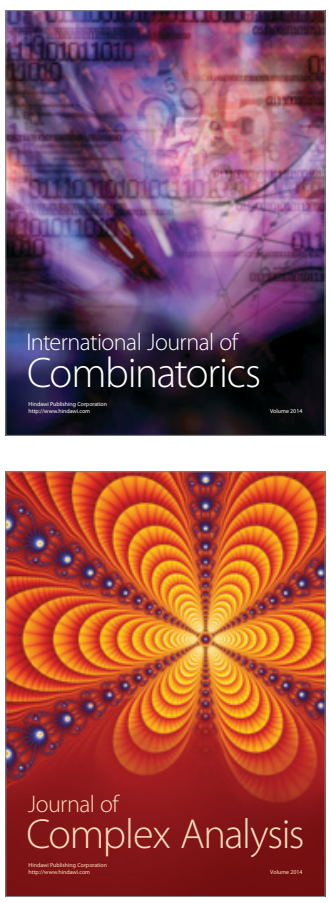

International Journal of

Mathematics and

Mathematical

Sciences
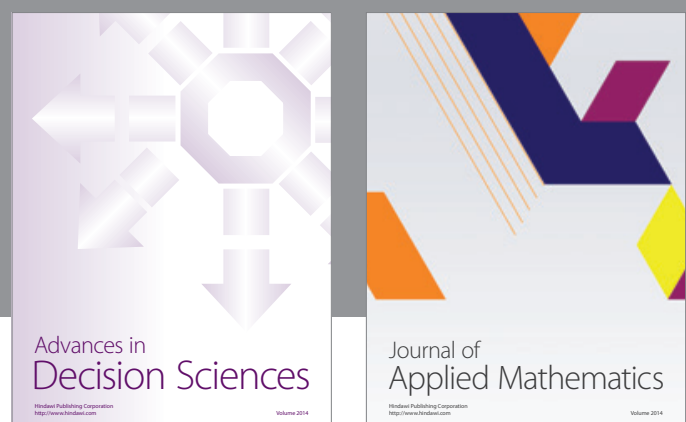

Journal of

Applied Mathematics
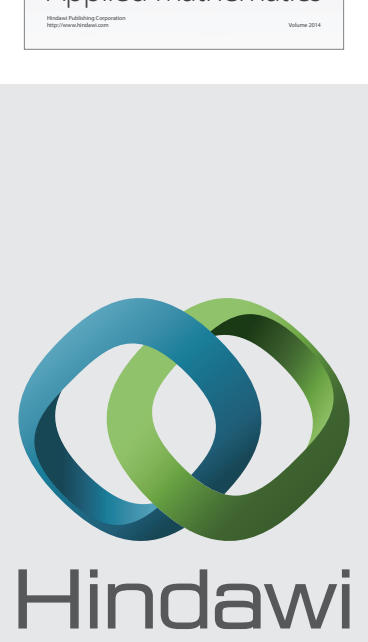

Submit your manuscripts at http://www.hindawi.com
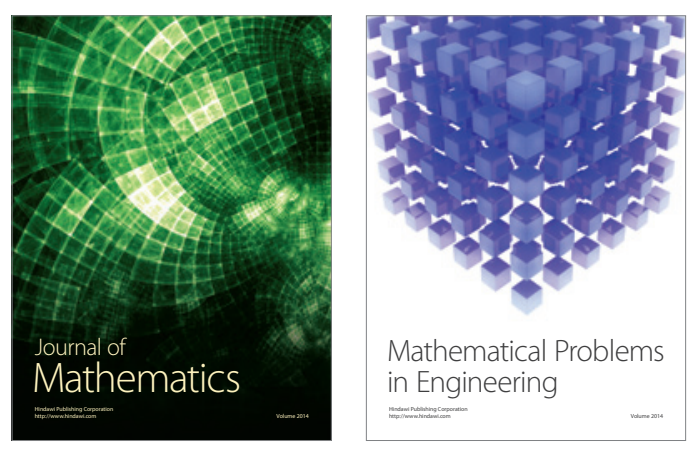

Mathematical Problems in Engineering
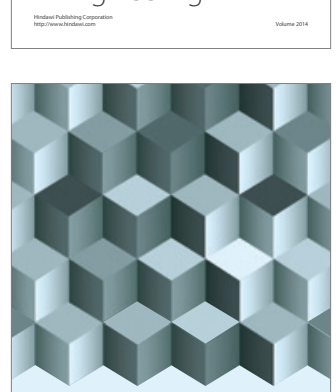

Journal of

Function Spaces
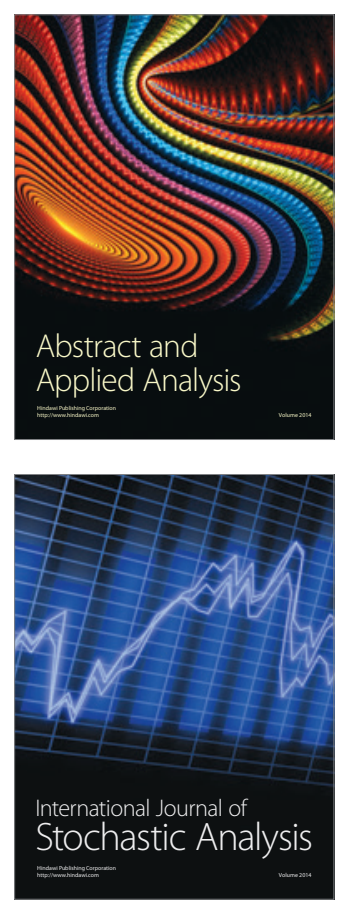

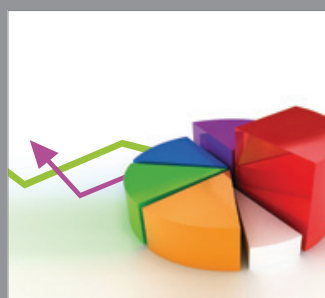

ournal of

Probability and Statistics

Promensencen
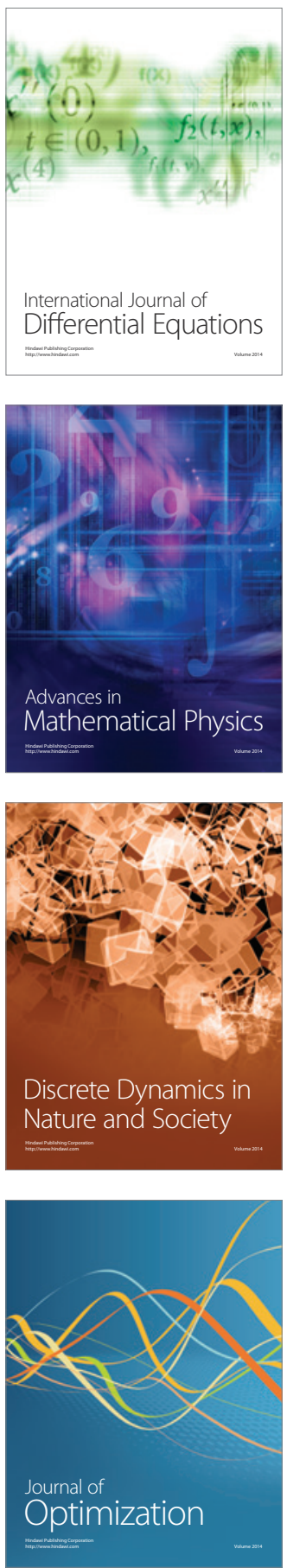